\title{
Use of antipsychotic and antidepressant within the Psychiatric Disease Centre, Regional Health Service of Ferrara
}

Stefano Bianchi*, Erica Bianchini and Paola Scanavacca

\begin{abstract}
Background: This study aimed at describing the type and dosage of psychopharmaceuticals dispensed to patients with psychiatric disorders and to assess the percentage of patients treated with antipsychotics and antidepressants, the associated therapies, treatment adherence, and dosages used in individuals registered at the Psychiatric Disease Center (PDC), Regional Health Service of Ferrara.

Methods: The analysis focused on therapeutic programmes presented to the Department of Pharmacy of the University Hospital of Ferrara of 892 patients treated by the PDC (catchment area of 134605 inhabitants). All diagnoses were made according to International Classification of Diseases (ICD-9). The analysis focused on prescriptions from September 2007 to June 2009. Data on adherence to prescribed therapy have were processed by analysis of variance.

Results: Among the patients 63\% were treated with antipsychotics and $40 \%$ with antidepressants. Among patients receiving antipsychotics $92 \%$ used second-generation antipsychotics (SGAs) whereas the remaining $8 \%$ used first generation antipsychotics (FGAs). Antipsychotic doses were lower than Daily Defined Dose (DDDs), and SGAs were often given with anticholinergics to decrease side effects. Mean adherence to antipsychotic therapy was $64 \%$. Among antidepressants, selective serotonin reuptake inhibitors (SSRIs) were the most often prescribed, 55\%. Dosages of these were within the limits indicated by the technical datasheet but higher than DDDs. Only 26\% of patients underwent monotherapy. In antidepressants polytherapy, medication was associated with another antidepressant, $6 \%$ or with an antipsychotic, 51\%. Mean adherence to the antidepressant therapy was $64 \%$.

Conclusions: Patients treated with antipsychotics tend to use doses lower than DDDs. The opposite tendency was noted in patients treated with antidepressants. Only a small percentage of patients (14\%) modified their neuroleptic therapy by increasing the dosage. On the contrary, patients treated with antidepressants mainly tended to reduce the doses of their drugs. This study highlights the tendency to follow combination therapies, prescribing SGAs together with anticholinergics in order to minimize extrapyramidal side effects or by combining two antidepressants. The study showed low adherence for both pharmaceutical therapies, which is typical in the setting of the analyzed diseases.
\end{abstract}

\section{Background}

Psychiatric disorders including different forms of depression and psychosis are highly debilitating conditions that generate strong discomfort in affected patients and a heavy burden on society as a whole; drugs indicated for these are expensive and treatment for these patients costs

\footnotetext{
* Correspondence: s.bianchi@ospfe.it
Department of Pharmacy, University Hospital of Ferrara, Corso Giovecca 203,

* Correspondence: s.bianchi@ospfe.it
Department of Pharmacy, University Hospital of Ferrara, Corso Giovecca 203, 44123 Ferrara (Italy
} 4h123 Ferrara (laly

more than the average cost of care for other common diseases such as diabetes and hypertension.

Several studies [1,2] demonstrated good efficacy of antipsychotics and antidepressants in alleviating mental disorders such as schizophrenia, depression, and bipolar disorder. However, the efficacy of controlling the symptoms of these disorders clearly depends on patient adherence to treatments, which is typically unsatisfactory [3]. 
Literature reports $[4,5]$ show significant differences between prescriptions in the clinical setting of psychiatric disorders and recommendations in official guidelines. In particular, studies on prescription of drugs have highlighted the frequent co-prescription of two or more medications [6-10], including anticholinergics [11,12], antidepressants, and antiepileptic drugs. Moreover, further studies [13] have shown that a high percentage of users receive drugs that affect the CNS above defined therapeutic ranges $[14,15]$.

In this study, we looked at the patterns of antipsychotic and antidepressant use within the Psychiatric Disease Centre (PDC), Regional Health Service of Ferrara, Italy.

\section{Method}

\section{Study design}

This was a descriptive, retrospective single-centre study focusing on drugs prescribed by the psychiatrists of the PDC, with a catchment area of 134605 inhabitants, and dispensed by the Department of Pharmacy of the University Hospital of Ferrara.

The study mainly focused on patients treated at the PDC with antipsychotics (Anatomical Therapeutic Chemical [ATC] N05) and antidepressants (ATC N06), even when associated with CNS-active drugs (ATC N). ATC is a classification system in which drugs are divided into different groups depending on the organ and their chemical, pharmacological, and therapeutic properties. Drug prescriptions including CNS-inactive drugs were not taken into account.

The observation period was 26 months from March 2007 to 31 May 2009. The number of patients evaluated during the evaluation period was 911 .

Since the diagnoses for $21 \%$ of patients were incomplete, it was not possible to obtain definitive diagnoses for all patients considered in the study. Furthermore, 19 patients (2\%) were ineligible for the study because they did not take drugs specified in the inclusion criteria mentioned above. Therefore, the total number of patients eligible for this study was 892 (98\%). Drugs prescribed to patients treated by the PDC were all dispensed by the hospital pharmacy.

\section{Subjects}

This study was approved by the Ethics Committee of Ferrara on 28/07/2011 and conducted by applying the legislative decree of June 24, 2003, no. 211 of Good Clinical Practice in clinical trials of medicines for clinical use. The study was conducted in accordance with the Declaration of Helsinki.

\section{Efficacy and safety assessment}

This study aimed to assess the use of antipsychotic agents (ATC N05) and antidepressants (ATC N06) dispensed to patients treated at the PDC, Regional Health Service of Ferrara. More specifically, it aimed to assess:

- clinical characteristics of patients;

- type of drugs prescribed and their dosage;

- doses used compared with those indicated by drug technical datasheets (Summary Product Characteristics; SPC) and the Daily Defined Dose (DDD) [16];

- potential modifications to therapy (change of active drugs and their doses or addition of other active drugs);

- percent patients treated with polytherapy and analysis of the most frequent combinations;

- patient adherence to prescribed therapies, calculated as percentage drugs consumed versus quantity prescribed.

\section{Description of instruments}

Patients treated at the PDC obtained drugs prescribed by psychiatrists from the Department of Pharmacy of the University Hospital of Ferrara. The pharmacist completed the dispensation database including the following information: patient data (name, date of birth); prescribing specialist; diagnosis (coded as ICD-9), prescription (limitation period; prescribed medication; dosage; pharmaceutical form; routes of administration; active substance or drug); and amount of medication dispensed calculated on the basis of previous drug dispensations.

From that database, we analyzed the following data: overview of patient data, pathology according to ICD-9, prescribing centre, number of tablets/capsules/vials distributed, duration of prescription, daily dosage, quantity consumed. All variations made by doctors on patients' prescriptions concerning routes and time of administration were also taken into account.

We calculated the minimum, average, and maximum doses of the psychopharmaceuticals used in therapies and compared them with the DDDs as well as the minimum and maximum dosages of typical and atypical drugs listed in the SPCs.

Being the only daily average dosage internationally recognized, the DDD value was chosen as a parameter to compare daily dosages of therapy. Moreover, the dosage corresponding to the maximum frequency used in therapy was calculated for each drug.

Patient level of adherence to prescribed therapies was also assessed, calculated as the ratio between the amount of drug they actually withdrew to that prescribed by the doctor.

\section{Statistics}

Data on adherence to prescribed therapy were assessed by analysis of variance (ANOVA). Adherence was 
reported as the mean and its confidence interval $95 \%$ to asses whether is a difference in adherence of the various molecules. For statistical analysis we used SPSS 18 software.

A t-test was performed on the average adherence of patients treated with FGAs compared with SGAs.

\section{Results}

\section{Patients}

A total of 892 patients were eligible for the study $(\mathrm{M} / \mathrm{F}$, 362/530); their clinical characteristics are shown in Table 1.

Mood disorders (especially major depressive disorder and bipolar disorder) and schizophrenia were the most frequently diagnosed conditions, accounting for $36 \%$ and $35 \%$, respectively, for a combined total of $72 \%$ of all diagnoses (Table 2).

\section{Antipsychotics}

In all, $63 \%$ of patients (564/892) were prescribed antipsychotics; $42 \%$ of them (235/564) were men and $58 \%$ (329/ $564)$ women. Their age range was $15-98$ years and most ( $40 \%$ of the total) were in the age range 41-60 years, although a few prescriptions were made to patients aged $<20$ years $(0.5 \%)$ and in their 90 s (1\%). Mean age of the analyzed population was 55 years $(\mathrm{SD} \% \pm 17)$.

\section{Type of prescribed drugs}

In patients on antipsychotics, 520/564 (92\%) took second-generation antipsychotics (SGAs) whereas the remaining $8 \%(44 / 564)$ took first-generation antipsychotics (FGAs). The distribution is shown in Table 3.

\section{Drug doses}

Mean doses were consistently lower than those indicated by the DDDs. Namely, drugs with prescribed doses slightly

Table 1 Features of patients eligible for the study

\begin{tabular}{lll}
\hline Parameter & $\mathbf{n}$ & $\%$ \\
\hline Men & 362 & 40.58 \\
Women & 530 & 59.42 \\
Age group & & \\
$10-20$ & 8 & 0.93 \\
$21-30$ & 50 & 5.61 \\
$31-40$ & 124 & 13.90 \\
$41-50$ & 190 & 21.30 \\
$51-60$ & 167 & 18.72 \\
$61-70$ & 154 & 17.26 \\
$71-80$ & 133 & 14.91 \\
$81-90$ & 61 & 6.84 \\
$91-100$ & 5 & 0.56 \\
Mean age, years & $56 \pm 10.09$ & \\
\hline
\end{tabular}

SD, standard deviation.
Table 2 Diagnosis reported in therapeutic programs and coded with the ICD-9 system

\begin{tabular}{lll}
\hline ICD- & Correspondence & $\%$ \\
\hline $\mathbf{9}$ & & $\mathbf{3 6 . 4}$ \\
$\mathbf{2 9 6}$ & Mood disorders & 35.4 \\
$\mathbf{2 9 5}$ & Schizophrenia & $\mathbf{5 . 6}$ \\
$\mathbf{2 9 8}$ & Non-organic psychosis & $\mathbf{4 . 7}$ \\
$\mathbf{2 9 9}$ & Pervasive developmental disorders & 4 \\
$\mathbf{2 9 7}$ & Delusional disorders & $\mathbf{3 . 9}$ \\
$\mathbf{2 9 0}$ & Dementia & $\mathbf{3}$ \\
$\mathbf{2 9 4}$ & Persistent mental illnesses caused by pathologic & \\
& conditions & $\mathbf{2 . 4}$ \\
$\mathbf{2 9 2}$ & Mental disorder caused by substances & $\mathbf{2}$ \\
$\mathbf{2 9 3}$ & Temporary mental illnesses caused by pathologic & \\
& conditions & $\mathbf{1 . 4 2}$ \\
$\mathbf{3 0 0}$ & Anxiety disorders & $\mathbf{0 . 8 5}$ \\
$\mathbf{3 0 9}$ & Adaptation reactions & $\mathbf{0 . 2 8}$ \\
$\mathbf{3 1 8 . 1}$ & Severe mental retardation & $\mathbf{0 . 1 4}$ \\
$\mathbf{3 0 7 . 1}$ & Anorexia nervosa & \\
\hline
\end{tabular}

ICD, International Classification of Diseases.

lower than the DDDs were: aripiprazole $13.2 \mathrm{mg}$ (DDD, 15 $\mathrm{mg}$ ); olanzapine $8.6 \mathrm{mg}$ (DDD, $10 \mathrm{mg}$ ); risperidone $3.7 \mathrm{mg}$ (DDD, $5 \mathrm{mg}$ ); and haloperidol $3.7 \mathrm{mg}$ (DDD, $8 \mathrm{mg}$ ) (Table 4). Drugs with prescribed doses much lower than the DDDs were: quetiapine $230.6 \mathrm{mg}$ (DDD, $400 \mathrm{mg}$ ); clozapine $179.5 \mathrm{mg}$ (DDD, $300 \mathrm{mg}$ ); clotiapine $38.6 \mathrm{mg}$ (DDD, $80 \mathrm{mg}$ ); levomepromazine $25 \mathrm{mg}$ (DDD, $300 \mathrm{mg}$ ); chlorpromazine $62.5 \mathrm{mg}$ (DDD, $300 \mathrm{mg}$ ) (Table 4).

Moreover, the following drugs were prescribed at doses lower than those described in the SPCs: clozapine $179.5 \mathrm{mg}$ (SPC, 200-450 mg); haloperidol $3.71 \mathrm{mg}$ (SPC, $60 \mathrm{mg}$ ); clotiapine $38.6 \mathrm{mg}$ (SPC, 100-120 mg); and levomepromazine $25 \mathrm{mg}$ (SPC 75-300 mg) (Table 4).

\section{Variations in therapy}

In all, $16 \%$ of the analyzed population (95/564 patients) changed their drug dosage during therapy: 44/564 patients (8\%) reduced it while $51 / 564$ patients (9\%) increased it. More specifically, increases in prescribed doses of the following drugs were noted: quetiapine $(24 / 51)$, olanzapine $(17 / 51)$, aripiprazole $(4 / 51)$, haloperidol $(3 / 51)$ and risperidone (3/51). Antipsychotics that were dose-reduced were olanzapine (25/44), aripiprazole (7/44), quetiapine (6/44), risperidone (5/44), and haloperidol (1/44).

The originally prescribed drug was switched to other medication in $14 \%$ of patients $(81 / 564)$. As such, the following antipsychotics were switched: olanzapine $0.8 \%$; risperidone $4 \%$; quetiapine $3 \%$; aripiprazole $1 \%$; clotiapine $0.4 \%$; haloperidol $0.4 \%$; and chlorpromazine $0.2 \%$.

\section{Concomitant medications used with antipsychotics}

In all, 427/564 patients (76\%) were treated with antipsychotics and other concomitant therapies. Concomitant 
Table 3 Patients treated with antipsychotics and treatment adherence

\begin{tabular}{|c|c|c|c|c|c|c|c|}
\hline \multirow[t]{2}{*}{ Drug } & \multirow[t]{2}{*}{$\mathrm{n}$} & \multirow[t]{2}{*}{$\%$ patients } & \multicolumn{3}{|c|}{ Adherence } & \multirow[t]{2}{*}{$\mathrm{Cl} 95 \%$} & \multirow[t]{2}{*}{ P-value } \\
\hline & & & Minimum & Mean & Maximum & & \\
\hline & \multicolumn{7}{|c|}{ SGAs } \\
\hline Aripiprazole & 43 & 7.6 & 7.78 & 62.07 & 100 & $22.6-80.1$ & 0.01 \\
\hline Olanzapine & 193 & 34.2 & 7.78 & 59.15 & 100 & $25.9-81.2$ & 0.01 \\
\hline Quetiapine & 156 & 27.7 & 8.33 & 66.68 & 100 & $27.9-88.5$ & 0.01 \\
\hline Risperidone & 109 & 19.3 & 8.33 & 59.75 & 100 & $29.3-84.3$ & 0.01 \\
\hline \multirow[t]{2}{*}{ Clozapine } & 19 & 3.4 & 7.8 & 51.80 & 100 & $29.7-85.2$ & 0.01 \\
\hline & \multicolumn{7}{|c|}{ FGAs } \\
\hline Haloperidol & 26 & 4.6 & 8.33 & 63.99 & 100 & $17.4-59.2$ & 0.01 \\
\hline Clotiapine & 8 & 1.4 & 16 & 66.38 & 100 & $24.3-96.5$ & 0.01 \\
\hline Levomepromazine & 6 & 1.1 & 33.33 & 68.06 & 100 & $32.8-87.9$ & 0.01 \\
\hline Clorpromazine & 4 & 0.7 & 22.22 & 69.44 & 100 & $20.4-80.0$ & 0.01 \\
\hline
\end{tabular}

SGA, second-generation antipsychotics;

FGA, first-generation antipsychotic.

therapies prescribed with antipsychotics included the following: antidepressants $40 \%$ (228/564); antiepileptics 16\% (88/564); anticholinergic 9\% (48/564); antiepileptic and antidepressant 7\% (40/564); anticholinergic and antidepressant 2\% (12/564); anticholinergic and antiepileptic 1\% (8/564); and anticholinergic, antidepressant, and antiepileptic $0.5 \%(3 / 564)$.

Among patients taking an antipsychotic with an antidepressant, $83 \%$ were on SGAs. Moreover, SGAs were frequently given with anticholinergics (73\%).

\section{Adherence to therapy}

Patient adherence to therapy is shown in Table 3; this parameter was calculated ranging from a minimum of $60 \%$ for risperidone to a maximum of $69 \%$ for chlorpromazine. Mean adherence to treatments was $64 \%(\mathrm{SD} \% \pm 28)$.

We used the statistical t-test to evaluate and compare the average value of first-generation antipsychotic adherence to that of the second generation. For FGAs, the adherence mean was $66.97(\mathrm{SD} \% \pm 2.35)$, while for SGAs it was $59.89(\mathrm{SD} \% \pm 5.37)$. This statistical testing

Table 4 Antipsychotic dosages in milligrams used in therapy

\begin{tabular}{|c|c|c|c|c|c|c|c|}
\hline \multirow[t]{2}{*}{ Drug } & \multirow[t]{2}{*}{ DDD } & \multicolumn{3}{|c|}{ Dosage } & \multirow[t]{2}{*}{ SPC } & \multirow{2}{*}{$\begin{array}{l}\text { Most commonly prescribed dosage, } \\
\text { mg }\end{array}$} & \multirow{2}{*}{$\begin{array}{l}\% \text { Patients with add-on } \\
\text { anticholinergic }\end{array}$} \\
\hline & & Minimum & Mean & Maximum & & & \\
\hline \multicolumn{8}{|c|}{ SGAs } \\
\hline Aripiprazole & 15 & 2.5 & 13.16 & 30 & $10-30$ & 10 & 2.3 \\
\hline Olanzapine & 10 & 1.3 & 8.6 & 20 & $5-20$ & 5 & 6.7 \\
\hline Quetiapine & 400 & 12.5 & 230.6 & 1200 & $\begin{array}{l}200- \\
800\end{array}$ & 200 & 2.6 \\
\hline Risperidone & 5 & 0.5 & 3.7 & 9 & $2-16$ & 2 & 11 \\
\hline Clozapine & 300 & 25 & 179.5 & 450 & $\begin{array}{l}200- \\
450\end{array}$ & 100 & 10.5 \\
\hline \multicolumn{8}{|c|}{ FGAs } \\
\hline Haloperidol & 8 & 1 & 3.71 & 8 & 60 & 2 & 27 \\
\hline Clotiapine & 80 & 14 & 38.6 & 100 & $\begin{array}{l}100- \\
120\end{array}$ & 50 & 87.6 \\
\hline Levomepromazine & 300 & 12.5 & 25 & 50 & $75-300$ & 25 & 0 \\
\hline Chlorpromazine & 300 & 62.5 & 62.5 & 100 & $30-75$ & 50 & 50.4 \\
\hline
\end{tabular}

Trascrizione fonetica

DDD, Defined Daily Dose;

SPC, Summary of Product Characteristics;

SGA, second-generation antipsychotic;

FGA, first-generation antipsychotics. 
(95\% confidence interval) resulted in a significant pvalue equal to.038, showing a difference of treatment adherence among patients receiving the two categories of antipsychotics, slightly higher for patients using FGAs compared with patients treated with SGAs.

\section{Antidepressants}

Among patients treated with psychopharmaceuticals, $40 \%(361 / 892)$ took antidepressants, among whom $63 \%$ $(228 / 361)$ were women and 37\% (133/361) men. Their mean age was 55 years $(\mathrm{SD} \% \pm 9)$. The most frequently observed age group was 41-60 years (43\%). However, a small number of prescriptions to patients aged 21-30 years $(5 \%)$ and $81-90$ years $(4 \%)$ were also made.

\section{Type of prescribed drugs}

Prescribed antidepressants are shown in Table 5. The most prescribed antidepressant was venlafaxine (24\%) whereas the most prescribed drug category was selective serotonin reuptake inhibitors (SSRIs) at 55\%.

\section{Drug doses}

Minimum, mean, and maximum dosages of antidepressants and their comparison versus the DDDs and the SPCs are displayed in Table 6. The following antidepressants were given at higher doses versus the DDD: citalopram $44.3 \mathrm{mg}$ (DDD, $20 \mathrm{mg}$ ); mirtazapine $44.2 \mathrm{mg}$ (DDD, $30 \mathrm{mg}$ ); paroxetine $29.5 \mathrm{mg}$ (DDD, $20 \mathrm{mg}$ ); sertraline $73.8 \mathrm{mg}$ (DDD, $50 \mathrm{mg}$ ); venlafaxine $129.2 \mathrm{mg}$ (DDD, $100 \mathrm{mg}$ ).

\section{Variations in therapy}

In all, 52 of 361 patients (14\%) modified the dose of their therapy: $56 \%(29 / 52)$ of patients reduced it, while the remaining $44 \%(23 / 52)$ increased it.

The following drugs were dose-decreased during the observation period: $19 / 361$ (5\%) cases for venlafaxine; $3 /$ 361 (0.8\%) each for citalopram and paroxetine; $2 / 361$
$(0.6 \%)$ for amitriptyline; and $1 / 361(0.3 \%)$ each for sertraline and clomipramine.

On the other hand, patients who increased their drug doses were: $10 / 361$ (3\%) for citalopram; 4/361 (1\%) for venlafaxine; $3 / 361(0.8 \%)$ for paroxetine; $2 / 361(0.6 \%)$ for sertraline; $1 / 361(0.3 \%)$ each for mirtazapine, fluoxetine, trazodone, and clomipramine.

In all, $6 \%$ of patients $(22 / 361)$ switched their originally prescribed medication to other drugs. More specifically, the following medications were switched to other drugs during the observation period: citalopram $23 \%$; mirtazapine $18 \%$, sertraline and venlafaxine both $14 \%$; trazodone, reboxetine, and fluoxetine all 9\%; and paroxetine 5\%.

\section{Concomitant therapies with antidepressants}

Only $26 \%$ of patients took antidepressants as monotherapy. Drugs taken in addition to antidepressants were: another antidepressant 6\% (22/361); antipsychotic 51\% (184/361); antipsychotic and antiepileptic 9\% (32/361); anticholinergic $0.5 \%$ (2/361); antiepileptic 4\% (13/361); two antipsychotics 3\% (11/361); antipsychotic and anticholinergic $0.3 \%(1 / 361)$; and antipsychotic, anticholinergic, and antiepileptic $0.3 \%(1 / 361)$.

\section{Mean adherence to therapy}

Mean adherence to therapy was: $81 \%$ for reboxetine; $77 \%$ for citalopram; $76 \%$ for trazodone; $59 \%$ for mirtazapine; $58 \%$ for paroxetine; $63 \%$ for sertraline; $62 \%$ for venlafaxine; and $52 \%$ for fluoxetine. Mean overall adherence to treatments was $64 \%$ (SD \% \pm 34$)$.

\section{Discussion}

\section{Antipsychotics}

Atypical antipsychotics were most frequently prescribed (92\%). Among atypical antipsychotics, olanzapine, quetiapine, and risperidone were the most prescribed, whereas among typical antipsychotics haloperidol was by far the most taken.

Table 5 Patients treated with antidepressants and treatment adherence

\begin{tabular}{cccccccc}
\hline Drug & $\mathbf{n}$ & \% patients & Minimum & $\begin{array}{c}\text { Adherence } \\
\text { Mean }\end{array}$ & Maximum & Cl 95\% & P-value \\
\hline Paroxetine & 75 & 20.83 & 7.78 & 57.7 & 100 & $23.8-76.5$ & 0.03 \\
Citalopram & 83 & 23.06 & 7.78 & 77.2 & 100 & $26.0-83.6$ & 0.03 \\
Venlafaxine & 87 & 24.17 & 8.33 & 61.65 & 100 & $24.1-81.2$ & 0.03 \\
Sertraline & 29 & 8.06 & 8.33 & 62.80 & 100 & $23.7-85.3$ & 0.03 \\
Mirtazapine & 32 & 8.89 & 59.14 & 59.14 & 100 & $25.8-82.2$ & 0.03 \\
Trazodone & 25 & 6.94 & 11.11 & 75.94 & 100 & $24.5-83.5$ & 0.03 \\
Fluoxetine & 10 & 2.78 & 13.33 & 52.18 & 93.33 & $18.9-73.1$ & 0.03 \\
Amitriptiline & 4 & 1.11 & 35.71 & 43.42 & 50 & $37.2-49.5$ & 0.03 \\
Clomipramine & 6 & 1.67 & 14.81 & 67.72 & 100 & $23.1-84.3$ & 0.03 \\
Reboxetine & 10 & 2.78 & 33.33 & 81.07 & 100 & $38.6-92.5$ & 0.03 \\
Total & $\mathbf{3 6 1}$ & & & & & &
\end{tabular}


Table 6 Antidepressants dosages in milligrams used in therapy

\begin{tabular}{|c|c|c|c|c|c|c|}
\hline \multirow[t]{2}{*}{ Drug } & \multirow[t]{2}{*}{ DDD } & \multicolumn{3}{|c|}{ Dosage } & \multirow[t]{2}{*}{ Most commonly prescribed dosage, mg } & \multirow[t]{2}{*}{ SPC } \\
\hline & & Minimum & Mean & Maximum & & \\
\hline Citalopram & 20 & 10 & 44.25 & 60 & 20 & $20-60$ \\
\hline Mirtazapine & 30 & 15 & 44.23 & 45 & 30 & $15-45$ \\
\hline Paroxetine & 20 & 5 & 29.54 & 60 & 40 & $20-60$ \\
\hline Sertraline & 50 & 30 & 73.82 & 180 & 100 & $50-200$ \\
\hline Venlafaxine & 100 & 37.5 & 129.2 & 300 & 150 & $75-375$ \\
\hline
\end{tabular}

Mean doses of atypical antipsychotics were within the limits indicated by the SPCs. When compared with the DDDs, although of limited use in such investigations, doses lower than the DDDs and within the limits indicated by the SPCs can mean two different things: on the one hand, low doses can be therapeutically successful, while on the other, their concomitant use allows the reduction of each individual drug dosage as well as of side effects. Moreover, we observed one case of a patient treated with quetiapine at a dosage $1200 \mathrm{mg} /$ day higher than that suggested in the technical datasheet (200-800 $\mathrm{mg})$.

Our analysis of typical neuroleptic daily dispensations showed that prescribed mean dosages were nearly all within the limits indicated by technical datasheets and the DDDs [17] except for two drugs. Once again, this suggests that therapeutic results are achievable at low dosages and that attempts are made to minimize side effects. The only exception here was chlorpromazine, for which maximum doses were higher than those indicated by technical datasheets and the DDDs.

The greatest dosage variations with antipsychotics during the therapy were seen for quetiapine and olanzapine.

The study showed that $14 \%(81 / 564)$ of patients on antipsychotics replaced the initial drug. More specifically, 74/81 (91\%) patients initially treated with SGAs were switched to other drugs; only $2 / 81$ (2\%) patients replaced the SGAs with FGAs whereas 6\% (5/81) of patients switched from FGAs to SGAs. The most often replaced drugs were olanzapine, risperidone, and quetiapine.

The most frequently noted combination therapy was an antipsychotic (mainly SGAs) and an anticholinergic.

SGA dosages used with anticholinergics were consistently lower than the DDDs. Since the risk of extrapyramidal side effects is very low when atypical drugs are taken, the combination use with anticholinergics would be unnecessary. However, the combined use of these drugs was very common and usually higher than FGAs. This therapeutic choice probably aims at further reducing the onset of side effects.
The majority of patients followed prescribed antipsychotic therapy [18] with a low adherence range of 51$66 \%$.

The difference in adherence to treatment (slightly higher among patients treated with FGAs compared with patients treated with SGAs) was statistically significant; however, we recognize the limits of the different types of patients in terms of disease severity. SGAs are currently the first choice of treatment; it often happens that FGAs are reserved to patients as a second choice or where SGAs are contraindicated. Probably for this reason, these patients are the most monitored by the specialists and this could be a reason for greater adherence to therapy for patients treated with FGAs.

In a study by Magliano et al. [6] carried out in 30 Italian mental health services which included 682 patients (M/F 469/213), the data, when compared with our results, revealed some differences. Their study included patients aged 18-60 years (mean age 37 years) and among them, $98 \%$ of the subjects used antipsychotics (63\% treated with FGAs and 35\% with SGAs). In our study, the recruited patients had a wider age range of 15-98 years (and therefore the average age was 55 years old) and only $63 \%$ used antipsychotic drugs, with a lower percentage of patients using FGAs (8\%) while 92\% were treated with SGAs. In our study, the use of combination therapy was more widespread than in the data obtained from the Magliano study, where only $5 \%$ of patients were prescribed an antipsychotic and an antidepressant (versus $40 \%$ of patients in Ferrara) and 7\% were receiving an antipsychotic and an anticholinergic (versus 9\% in Ferrara).

\section{Antidepressants}

The most prescribed antidepressant was venlafaxine whereas the most prescribed category of antidepressants, $55 \%$ of all antidepressants, was the SSRI class. These data confirm the results highlighted by other studies [19], which showed that SSRIs are the most prescribed antidepressants in Italy. This figure has also been confirmed by other European studies such as those by Finder [19] and Serna [20]. Our analysis shows that doses 
were almost always within the limits indicated by the technical sheets but consistently above those indicated by the DDDs.

During the therapy, a small proportion (14\%) of patients modified their dosage of prescribed drug: $8 \%$ of them reduced it while the remaining $6 \%$ increased it. The most frequently up-titrated antidepressant was citalopram (19\% of the total of patients who increased their therapy) followed by venlafaxine (8\%) and paroxetine (6\%). On the other hand, it emerged that the prescribed dosage of venlafaxine was usually reduced (in $37 \%$ of patients).

Citalopram was apparently not very efficacious based on our finding that it was dose-increased more than other drugs despite having a good adherence.

In most cases, antidepressants were prescribed concomitantly with an antipsychotic or another antidepressant, suggesting quite a high comorbidity of depression and psychosis in patients in this study. The presence of a number of patients taking two antidepressants concomitantly also suggests that depression symptoms are difficult to treat successfully with just a single drug.

\section{Conclusion}

This retrospective study evaluated the prescription profiles of 892 patients treated by the PDC. SGAs were the most commonly prescribed antipsychotic agents. Dosages prescribed were usually within those recommended by the drug manufacturers and international standards. These agents were often used with concomitant therapies, especially with anticholinergics, despite the reduced risk of extrapyramidal side effects. SSRIs were the most commonly prescribed antidepressants, at dosages within the range recommended by suppliers but higher than the DDDs. Antidepressants were mainly prescribed in association with a second antidepressant or an antipsychotic. For both classes of drugs, we observed an average level of patient adherence to their therapies, although there have not been many studies that have investigated adherence to therapy of antipsychotics and antidepressants. Unfortunately, this may represent a limitation to our study because it does not allow comparison of our results to those obtained in other populations.

\footnotetext{
List of abbreviations

ATC: Anatomical Therapeutic Chemical; PDC: Psychiatric Disease Centre; SPC: Summary Product Characteristic; DDD: Daily Defined Dose; ICD-9: International Statistical Classification of Diseases and Related Health Problems; SD: standard deviation; SSRI: selective serotonin reuptake inhibitor; SGA: second-generation antipsychotic; FGA: first-generation antipsychotic.
}

\section{Acknowledgements}

The authors thank the staff of the mental health centre and the health statistics service at the University Hospital of Ferrara, whose assistance allowed the realization of this study.

\section{Authors' contributions}

SB defined the design of this study, establishing the number of patients to be enrolled, the number of prescriptions by therapeutic analysis, and the type of data needed to achieve the defined objectives. SB submitted the study to the Ethics Committee of Ferrara, established the protocols for statistical data analysis, continuously monitored the data obtained, and contributed to the writing and revision of the article. SB defined operating modes, introducing the DDD for comparison of treatments in the dosage given. EB analyzed the requirements of patients and processed the data to obtain patient characteristics, types of drugs prescribed, dosages used in therapy, changes of drug or dosing and possible associations. He participated in drafting and revising the article.

PS evaluated the data obtained and the execution of the study, also participating in the drafting and revision of the article.

All authors read and approved the final manuscript.

\section{Competing interests}

The authors declare that they have no competing interests.

Received: 9 April 2011 Accepted: 20 December 2011

Published: 20 December 2011

\section{References}

1. Hirsch SR, Kissling W, Bauml J, Power A, O'Connor R: A 28-week comparison of ziprasidone and haloperidol in outpatients with stable schizophrenia. J Clin Psychiatriy 2002, 63:516-523.

2. Kane JM, Carson WH, Saha AR, et al: Efficacy and safety of aripiprazole and haloperidol versus placebo in patient with schizophrenia and schizoaffective disorder. J Clin Psychiatriy 2002, 63:763-771.

3. Ren XS, Herz L, Qian S, Smith E, Kazis LE: Measurement of treatment adherence with antipsychotic agents in patient with schizophrenia Neuropsychiatric Disease and Treatment 2009, 5:491-498.

4. American Psychiatric Association: Practice Guideline for the Treatment Of Patients With Schizophrenia. American Psychiatric Press, Washington DC; 2007.

5. National Institute for Clinical Excellence-Nice: Schizophrenia-Full National Clinic Guideline On Core Intervention In Primary And Secondary Care. Gaskell Press e British Psychological Society, London; 2003.

6. Magliano L, Fiorillo A, Guarneri M, Marasco C, De Rosa C, Malangone C, Maj M: Prescription of psychotropic drugs to patients with schizophrenia: an Italian national survey. Eur J Clin Pharmacol 2004, 60:513-522.

7. Schmidt LG, Lammers V, Stockel M, Muller-Oerlinghausen B: Recent trends in prescribing psychotropic drugs at a psychiatric university hospital 1981-1984. Pharmacopsychiatry 1998, 21:126-130.

8. Tognoni G: Pharmacoepidemiology of psychotropic drugs in patients with severe mental disorders in Italy: Italian Collaborative Study Group on the Outcome of Severe Mental Disorders. Eur J Clin Pharmacol 1999, 55:685-690.

9. Williams $\mathrm{CL}$, Johnstone BM, Kesterson JG, Yavor KA, Schmetzer AD: Evaluation of antypsychotics and concomitance medication use patterns in patients with schizophrenia. Care Med 1999, 4:AS81-AS86.

10. Brunot A, Lachaux B, Sontag H, Casadebaig F, Philippe A, Rouillon F, CleryMelin P, Hergueta T, Llorca PM, Moreaudefarges T, Guillon P, Lebrun T: Pharmaco-epidemiological study on antipsychotic drug prescription: in French psychiatry: patient characteristics, antyphichotic treatment and care management for schizophrenia. Encephale 2002, 28:129-138.

11. Keks NA, Altson K, Hope J, Krapivensky N, Culhane C, Tanaghow A, Doherty P, Bootle A: Use of antipsychotics and adjunctive medications by an inner urban community psychiartic service. Aust NZJ Psychiatriy 1999, 33:896-901.

12. Yip KC, Ungvari GS, Cheung HK, Ng FS, Lau ST: A survey of antipsychotic treatment for schizophrenia in Hong Kong. Chin Med J 1997, 110:792-796.

13. Lesli DL, Rosenheck RA: Use of pharmacy date to assess quality of pharmacotherapy for schizophrenia in a national health care system: individual and facility predictors. Med Care 2001, 39:907-907.

14. Lehman AF, Steinwachs DM, the Co-Investigators of the Port Project: Translating research into practice: the schizophrenia Patient Outcomes Research Team (PORT) treatment recommendations. Schizophr Bull 1998, 24:1-10. 
15. Bardui C, Danese A, Guaiana G, Mapelli L, Miele L, Monzani E, Percudani M, on behalf of the study group: Prescribing second generation antipsychotics and the evolving standard of care in Italy. Phacopsychiatry 2002, 35:239-243.

16. WHO Collaborating center for Drug Statistics Methodology. Guidelines for ATC classification and DDD assignment 2010 Oslo; 2009.

17. Compendio Farmaceutico Ospedaliero. Farmadati Italia; 2010, Updating n - 97, .

18. Ren XS, Herz L, Qian S, Smith E, Kazis LE: Measurement of treatment adherence with antipsychotic agents in patient with schizophrenia. Neuropsychiatric Disease and Treatment 2009, 5:491-498.

19. Bauer M, Monz BU, Montejo AL, Quail D, Dantchev N, Demyttenaere K, Garcia CA, Grassi L, Perahia DGS, Reed C, Tylee A: Prescribing patterns of antidepressants in Europe: results from the Factors Influencing Depression Endpoints Research (FINDER) study. European Psychiatry 2008, 23:66-73.

20. Serna MC, Cruz I, Real J, Gascò E, Galvan L: Duration and adherence of antidepressant treatment (2003 to 2007) based on prescription database. European Psychiatry 2009, 7-12.

\section{Pre-publication history}

The pre-publication history for this paper can be accessed here: http://www.biomedcentral.com/1472-6904/11/21/prepub

doi:10.1186/1472-6904-11-21

Cite this article as: Bianchi et al:: Use of antipsychotic and

antidepressant within the Psychiatric Disease Centre, Regional Health

Service of Ferrara. BMC Clinical Pharmacology 2011 11:21.

\section{Submit your next manuscript to BioMed Central} and take full advantage of:

- Convenient online submission

- Thorough peer review

- No space constraints or color figure charges

- Immediate publication on acceptance

- Inclusion in PubMed, CAS, Scopus and Google Scholar

- Research which is freely available for redistribution

Submit your manuscript at www.biomedcentral.com/submit 